\section{A gravitationally lensed quasar with quadruple images separated by 14.62 arcseconds}

Naohisa Inada ${ }^{1}$, Masamune Oguri ${ }^{1}$, Bartosz Pindor $^{2}$, Joseph F. Hennawi ${ }^{2}$, Kuenley Chiu ${ }^{3}$, Wei Zheng ${ }^{3}$, Shin-Ichi Ichikawa ${ }^{4}$, Michael D. Gregg ${ }^{5,6}$, Robert H. Becker ${ }^{5,6}$, Yasushi Suto ${ }^{1}$, Michael A. Strauss ${ }^{2}$, Edwin L. Turner ${ }^{2}$, Charles R. Keeton ${ }^{7}$, James Annis ${ }^{8}$, Francisco J. Castander ${ }^{9}$, Daniel J. Eisenstein ${ }^{10}$, Joshua A. Friemann ${ }^{7,8}$, Masataka Fukugita ${ }^{11}$, James E. Gunn ${ }^{2}$, David E. Johnston ${ }^{7}$, Stephen M. Kent ${ }^{8}$, Robert C. Nichol ${ }^{12}$, Gordon T. Richards ${ }^{2}$, Hans-Walter Rix ${ }^{13}$, Erin Scott Sheldon ${ }^{7}$, Neta A. Bahcall ${ }^{2}$, J. Brinkmann ${ }^{14}$, Željko Ivezić ${ }^{2}$, Don Q. Lamb ${ }^{7}$, Timothy A. McKay ${ }^{15}$, Donald P. Schneider ${ }^{16}$ \& Donald G. York ${ }^{7,17}$

${ }^{1}$ Department of Physics, School of Science, The University of Tokyo, 113-0033, Japan

${ }^{2}$ Princeton University Observatory, Peyton Hall, Princeton, New Jersey 08544, USA

${ }^{3}$ Department of Physics and Astronomy, Johns Hopkins University,

3701 San Martin Drive, Baltimore, Maryland 21218, USA

${ }^{4}$ National Astronomical Observatory, 2-21-1 Osawa, Mitaka, Tokyo 181-8588, Japan

${ }^{5}$ Department of Physics, University of California at Davis, 1 Shields Avenue,

Davis, California 95616, USA

${ }^{6}$ Institute of Geophysics and Planetary Physics, Lawrence Livermore National

Laboratory, L-413, 7000 East Avenue, Livermore, California 94550, USA

${ }^{7}$ Department of Astronomy and Astrophysics, University of Chicago,

5640 South Ellis Avenue, Chicago, Illinois 60637, USA

${ }^{8}$ Fermi National Accelerator Laboratory, PO Box 500, Batavia, Illinois 60510,

USA

${ }^{9}$ Institut d'Estudis Espacials de Catalunya/CSIC, Gran Capita 2-4,

08034 Barcelona, Spain

${ }^{10}$ Steward Observatory, University of Arizona, 933 North Cherry Avenue, Tucson, Arizona 85721, USA

${ }^{11}$ Institute for Cosmic Ray Research, The University of Tokyo, 5-1-5 Kashiwa,

Chiba 277-8582, Japan

${ }^{12}$ Department of Physics, Carnegie Mellon University, Pittsburgh,

Pennsylvania 15213, USA

${ }^{13}$ Max-Planck Institute for Astronomy, Königstuhl 17, D-69117 Heidelberg, Germany

${ }^{14}$ Apache Point Observatory, PO Box 59, Sunspot, New Mexico 88349, USA ${ }^{15}$ Department of Physics, University of Michigan, 500 East University Avenue, Ann Arbor, Michigan 48109, USA

${ }^{16}$ Department of Astronomy and Astrophysics, Pennsylvania State University, 525 Davey Laboratory, University Park, Pennsylvania 16802, USA

${ }^{17}$ Enrico Fermi Institute, University of Chicago, 5640 South Ellis Avenue, Chicago, Illinois 60637, USA

Gravitational lensing is a powerful tool for the study of the distribution of dark matter in the Universe. The cold-darkmatter model of the formation of large-scale structures (that is, clusters of galaxies and even larger assemblies) predicts $^{1-6}$ the existence of quasars gravitationally lensed by concentrations of dark matter ${ }^{7}$ so massive that the quasar images would be split by over 7 arcsec. Numerous searches ${ }^{8-11}$ for large-separation lensed quasars have, however, been unsuccessful. All of the roughly 70 lensed quasars known ${ }^{12}$, including the first lensed quasar discovered $^{13}$, have smaller separations that can be explained in terms of galaxy-scale concentrations of baryonic matter. Although gravitationally lensed galaxies ${ }^{14}$ with large separations are known, quasars are more useful cosmological probes because of the simplicity of the resulting lens systems. Here we report the discovery of a lensed quasar, SDSS J1004+4112, which has a maximum separation between the components of 14.62 arcsec. Such a large separation means that the lensing object must be dominated by dark matter. Our results are fully consistent with theoretical expectations ${ }^{3-5}$ based on the cold-dark-matter model.
For bright quasars (i-band magnitude $i<19$ ), the probability of gravitational lensing ${ }^{15}$ is only about $0.1 \%$; the majority of these lenses have small separations, due to a single massive galaxy. The fraction of large-separation lensed quasars is predicted to be $0.01 \%$ or less ${ }^{3-5}$; thus it is not surprising that none have been found to date $^{8-11}$. In order to find such objects, we need samples of tens of thousands of quasars, such as those generated by the Sloan Digital Sky Survey ${ }^{16,17}$ (SDSS). The SDSS is conducting both a photometric survey $^{18-22}$ using five broad optical bands ${ }^{22}(\mathrm{u}, \mathrm{g}, \mathrm{r}, \mathrm{i}$ and $\mathrm{z}$ ) and a spectroscopic survey ${ }^{23}$ of 10,000 square degrees of the sky centred approximately on the North Galactic Pole, using a dedicated widefield 2.5-m telescope at the Apache Point Observatory.

We searched for large-separation lensed quasars in a sample of $\sim 29,500$ spectroscopically confirmed SDSS quasars ${ }^{24}$ at redshifts $z$ of 0.6-2.3, a sample roughly three times larger than those used in previous searches. Even with this large sample, the expected number of large-separation lensed quasars is of the order of unity. In the field around each quasar in the sample, we searched for stellar objects with colours differing by less than 0.1 from those of the quasar, with separations between $7.0^{\prime \prime}$ and $60.0^{\prime \prime}$ and with flux greater than onetenth that of the quasar. SDSS J1004+4112 was identified as a 'quadruple' large-separation lensed quasar candidate using these criteria. Only one of the four components (component B, see below) has an SDSS spectrum (the SDSS hardware ${ }^{23}$ does not allow pairs of objects separated by less than $55^{\prime \prime}$ to be observed on a single plate), and therefore, we obtained spectra of all four components using the

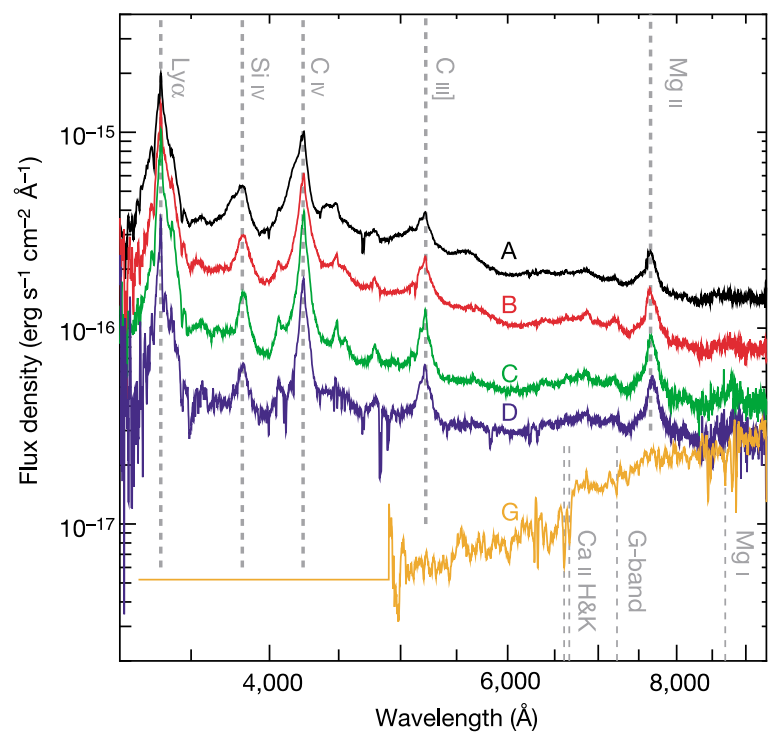

Figure 1 The Keck spectra of the four quasar components $A-D$, and the brightest galaxy $G$ in the lensing cluster. See Fig. 2 for these identifications (A-D, and G). The data were taken using the Low-Resolution Imaging Spectrometer ${ }^{29}$ of the Keck I telescope. The exposure times were $900 \mathrm{~s}$ for each component. The dispersion is $1.09 \AA$ pixel $^{-1}$. The data were reduced in a standard method using IRAF (IRAF is the image reduction and analysis facility, distributed by the National Optical Astronomy Observatories). The black solid line, the red solid line, the green solid line and the blue solid line represent the spectra of components $A, B, C$ and $D$, respectively. The vertical grey dashed lines with labels at the top of the figure $(3,323.6 \AA, 3,818.7 \AA$, 4,235.1 $\AA$, 5,218.5 $\AA$ and $7,651.8 \AA)$ represent the positions of emission lines of the respective ions redshifted to $z=1.734$ of Lyo $(1,215.67 \AA)$, Si Iv $(1,396.76 \AA)$, C Iv $(1,549.06 \AA)$, C III] $(1,908.73 \AA)$ and Mg ॥ $(2,798.75 \AA)$, respectively. All emission lines are clearly at the same redshift. The orange solid line represents the Keck spectrum of component $G$ at the same dispersion. The exposure time was also $900 \mathrm{~s}$ for component $\mathrm{G}$. The vertical thinner grey dashed lines with labels at the lower right of the figure $(6,608.2 \AA, 6,666.7 \AA, 7,231.0 \AA$, and $8,694.0 \AA)$ represent the positions of absorption lines of the respective ions redshifted to $z=0.680$ of Ca ॥ H\&K (3,933.7 $\AA$ and 3,968.5 $\AA$ ), G-band (4,304.4 $\AA$ ), and Mg I B-band (5,175.3 $\AA$ ), respectively. There are no data below $\sim 4,900 \AA$ in the spectrum of component $\mathrm{G}$. 


\begin{tabular}{|c|c|c|c|c|c|c|c|}
\hline \multicolumn{8}{|c|}{ Table 1 Astrometry and photometry for SDSS J1004+4112 } \\
\hline Object & $\mathrm{RA}(\mathrm{h} \min \mathrm{s})^{*}$ & Dec. $\left({ }^{\prime}{ }^{\prime \prime \prime}\right)^{*}$ & $g \dagger$ & $r \dagger$ & i† & $z \dagger$ & $\Delta \theta \neq$ \\
\hline A & 100434.794 & +411239.29 & $18.67 \pm 0.03$ & $18.70 \pm 0.02$ & $18.46 \pm 0.02$ & $18.43 \pm 0.05$ & $3.73^{\prime \prime}$ \\
\hline $\mathrm{B}$ & 100434.910 & +411242.79 & $19.05 \pm 0.06$ & $19.10 \pm 0.06$ & $18.86 \pm 0.06$ & $18.92 \pm 0.06$ & $0.00^{\prime \prime}$ \\
\hline C & 100433.823 & +411234.82 & $19.71 \pm 0.03$ & $19.73 \pm 0.02$ & $19.36 \pm 0.03$ & $19.31 \pm 0.07$ & $14.62^{\prime \prime}$ \\
\hline $\mathrm{D}$ & 100434.056 & +411248.95 & $20.67 \pm 0.04$ & $20.51 \pm 0.04$ & $20.05 \pm 0.04$ & $20.00 \pm 0.13$ & $11.44^{\prime \prime}$ \\
\hline $\mathrm{G}$ & 100434.170 & +411243.66 & $22.11 \pm 0.40$ & $20.51 \pm 0.13$ & $19.54 \pm 0.09$ & $19.04 \pm 0.21$ & $8.44^{\prime \prime}$ \\
\hline
\end{tabular}

${ }^{*}$ RA, right ascension; dec., declination. RA and dec. are given in the J2000 system. These celestial coordinates were measured on the basis of the celestial coordinates of component B. The positional errors of components A, C and D (not including the absolute positional errors of component B) are $0.01^{\prime \prime}$ and that of component $\mathrm{G}$ is $0.05^{\prime \prime}$ per coordinate.

$\dagger g, r, i$ and $z$ mean the magnitudes of each band.

$\ddagger$ Separation angles relative to component $\mathrm{B}$.

Keck I telescope at the W. M. Keck Observatory. The results are shown in Fig. 1. All four components indeed show quasar-like features, with all emission lines giving a consistent redshift $z=1.734 \pm 0.002$; the velocity differences of the quasar components are $\sim 100 \mathrm{~km} \mathrm{~s}^{-1}$, comparable to the observational uncertainty. Although it is not obvious from Fig. 1, there are CIV absorption line systems at $z=1.732$ in each of the four quasar spectra; this is an absorption system associated with the quasar itself, further supporting the lensing hypothesis: the four quasar images are from the same physical source. The differences in their spectra may be explained by the modest time-variability of the source quasar over $\sim 1 \mathrm{yr}$, the expected gravitational lensing timedelay $^{25}$ among those different images.

Additional strong support for the lensing hypothesis comes from the identification of the galaxy cluster responsible for the largeseparation lensing. From the observed image separations (the maximum separation is $14.62^{\prime \prime}$, between images B and C), we infer that the lensing object should have a velocity dispersion in

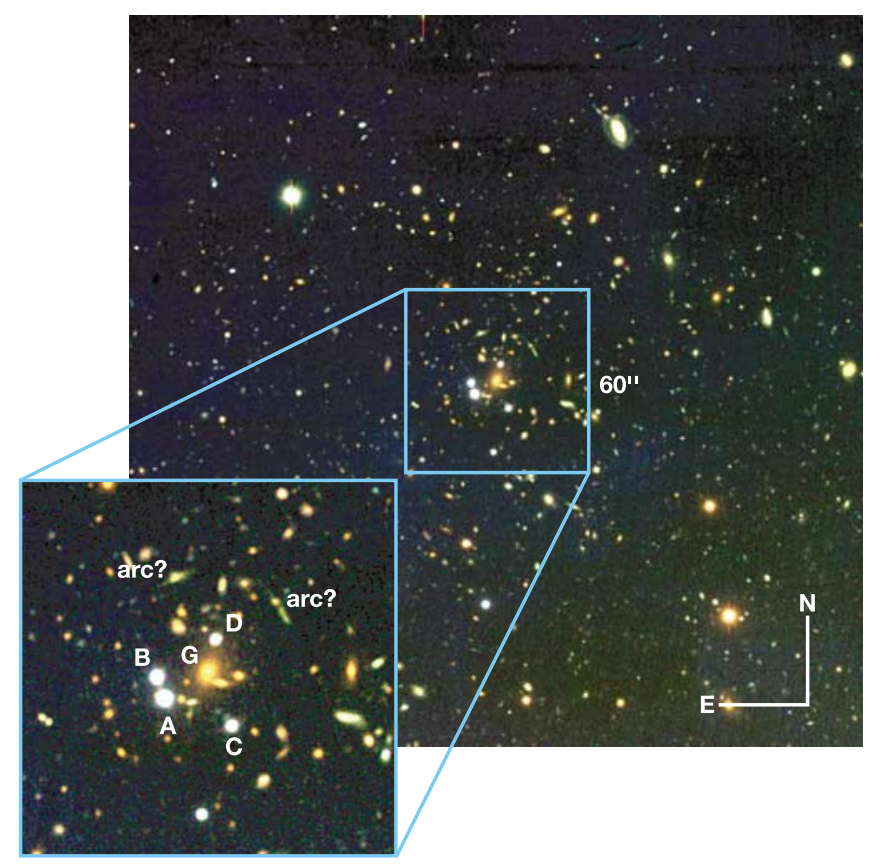

Figure 2 The gri composite Subaru image of the field around SDSS J1004+4112. The data were taken using the Subaru Prime Focus Camera ${ }^{30}$ of the Subaru telescope. The magnitude limit is $i \approx 26.0$. The central $60^{\prime \prime}$ square is shown in an expanded view. The four quasar components are marked as $A, B, C$ and $D$, and the bright galaxy located between the four quasar components is marked as $\mathrm{G}$. The separation between components $A$ and $D$ is $12.77^{\prime \prime}$, and that between components $B$ and $C$ is $14.62^{\prime \prime}$. The positions (J2000) and the magnitudes of the components A-D and the brightest galaxy (component $\mathrm{G}$ ) between the four quasar components are summarized in Table 1. Many faint galaxies can be seen-their positions and colours are consistent with being members of a cluster $(z=0.68)$ centred on component $G$. Two possible arclets (marked as 'arc?') can also be seen. The seeing had a full-width at half-maximum of $0.6^{\prime \prime}$. excess of $600 \mathrm{~km} \mathrm{~s}^{-1}$. Thus the lensing object cannot be a single galaxy, but must be rather a group or cluster of galaxies that has a sufficiently concentrated distribution of dark matter. To identify the lensing object, we obtained deep optical images of the system using the Subaru telescope of the National Astronomical Observatory, Japan. The result is shown in Fig. 2. A number of galaxies are clearly detected around component G, suggesting that it is the most luminous galaxy of the cluster. We obtained a spectrum of component $\mathrm{G}$ using the Keck I telescope. The spectrum shows a number of absorption features characteristic of a early-type galaxy at a

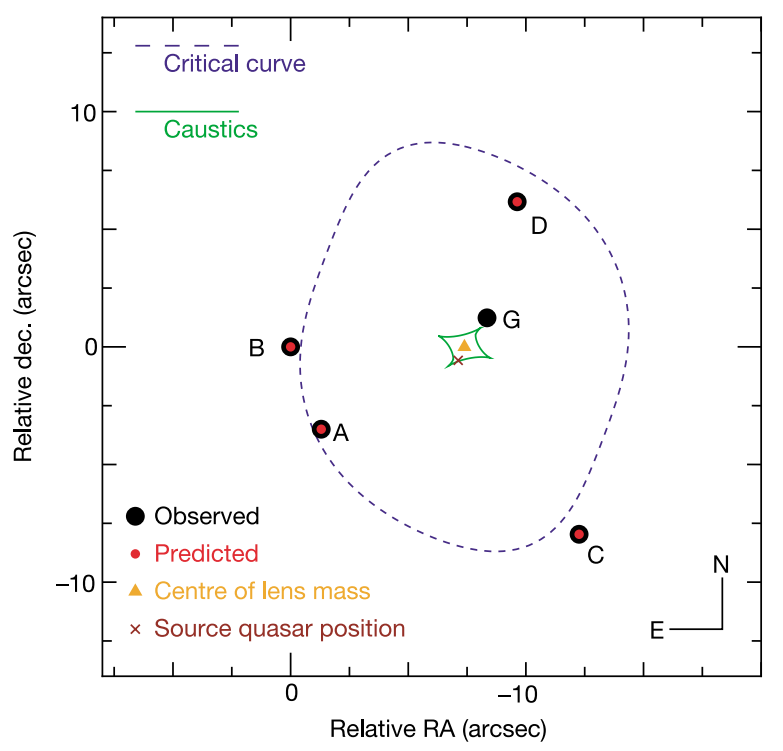

Figure 3 The best-fit lens model prediction compared with the observation. We used a lensing model of a singular isothermal ellipsoid (SIE) with external shear. The best-fit model has an Einstein radius of the SIE model $\alpha_{\mathrm{e}}=6.906$ " (corresponding to a velocity dispersion $\sim 700 \mathrm{~km} \mathrm{~s}^{-1}$ at the cluster redshift), magnitude and position angle of the shear $\gamma=0.250$ and $\theta_{\gamma}=-60.925^{\circ}$ (measured east of north), and ellipticity and its position angle $e=0.498$ and $\theta_{e}=21.434^{\circ}$ (measured east of north), with a source quasar position $(\Delta \mathrm{RA}, \Delta$ dec. $)=\left(-7.124^{\prime \prime},-0.574^{\prime \prime}\right)$ and a centre of lensing mass $(\Delta \mathrm{RA}, \Delta$ dec. $)=\left(-7.387^{\prime \prime},-0.004^{\prime \prime}\right)$ relative to the centre of component $\mathrm{A}$. The black filled circles represent the observed positions of components A, B, C, D and G, and the red filled circles represent the predicted positions of components $A-D$. The green solid line is the position of the caustic in the source plane, and the blue dashed line represents the critical curve in the image plane. The small brown filled cross is the predicted position of the source quasar, and the small orange filled triangle is the predicted position of the centre of the lens mass. The differences between the observed and modelled image positions are much smaller than the observational uncertainties. The flux ratios predicted from the model, B/A, C/A and D/A are $0.78,0.43$ and 0.22 , respectively. The total magnification of the quasar images predicted by the model is 56.48 . The predicted flux ratios are close to the observational results; $B / A=0.69 \pm 0.04, C / A=0.46 \pm 0.02$ and $\mathrm{D} / \mathrm{A}=0.25 \pm 0.01$ (measured from the $\mathrm{i}$-band image). Microlensing by substructures and/or reddening by the Mg $\|$ absorption line systems that are seen in each spectrum might be the cause of the differences between the predicted flux ratios and the observations. 
redshift of $z=0.6799 \pm 0.0001$. We also obtained spectra of two faint galaxies immediately to the southwest of component G (Fig. 2) using the Faint Object Camera and Spectrograph ${ }^{26}$ of the Subaru telescope. The redshifts of these two faint galaxies are $z=0.6751 \pm 0.0001$, strongly suggesting a cluster of galaxies at $z \approx 0.68$ centred on component $\mathrm{G}$. Clusters are dominated by elliptical galaxies, which all have very similar spectral energy distributions.

Many of the faint galaxies in Fig. $2(\sim 40$ galaxies around component $\mathrm{G}$ ) have colours that are similar to that of component $\mathrm{G}$. The colours are consistent with the expected colours of elliptical galaxies at $z \approx 0.68(g-r \approx 1.8$ and $r-i \approx 1.1)$. In addition, there is an X-ray source in this direction detected by the ROSAT All-Sky Survey ${ }^{27}$ ( 0.236 counts per second in a 473-s exposure). The emission, however, comes most probably from the quasar, because the detected X-ray flux is too strong for typical clusters of galaxies at $z=0.68$. Finally, we note two possible arclets (highly distorted images of background galaxies due to gravitational lensing) in Fig. 2 (marked as 'arc?') close to component D. If future observations confirm that the arclets are indeed lensed background galaxies, they will provide strong additional constraints on the total mass distribution of the lensing cluster.

The lensing interpretation is further supported by a theoretical model of SDSS J1004+4112. We fitted the positions of the four quasar components with a singular isothermal ellipsoid (SIE) plus external shear model using lens modelling software ${ }^{28}$. The best-fit model is illustrated in Fig. 3. The positions and relative brightnesses of all components agree well with the lens model predictions. The centre of the lensing mass is offset from the centre of component $\mathrm{G}$ by about $10 \mathrm{kpc}$ at the cluster redshift, but the brightest galaxy of a cluster is not always found exactly at the centre of the potential well of that cluster.

The identical redshifts $(z=1.734)$ and the spectral energy distributions of the four lensed components, the existence of a lensing cluster of galaxies $(z=0.68)$, and the presence of possible arclets confirm the hypothesis that the quasar is lensed by this cluster. Furthermore, a theoretical lensing model involving the cluster and external shear simultaneously accounts for the observed geometry of the system and the relative brightness of the images. The present work represents the discovery of a long-predicted but previously undetected population of large-separation lensed quasars.

Received 30 July; accepted 23 October 2003; doi:10.1038/nature02153.

1. Narayan, R. \& White, S. D. M. Gravitational lensing in a cold dark matter universe. Mon. Not R. Astron. Soc. 231, 97-103 (1988).

2. Wambsganss, J., Cen, R., Ostriker, J. P. \& Turner, E. L. Testing cosmogonic models with gravitational lensing. Science 268, 274-276 (1995).

3. Keeton, C. R. \& Madau, P. Lensing constraints on the cores of massive dark matter halos. Astrophys. J. 549, L25-L28 (2001)

4. Wyithe, J. S. B., Turner, E. L. \& Spergel, D. N. Gravitational lens statistics for generalized NFW profiles: Parameter degeneracy and implications for self-interacting cold dark matter. Astrophys. J. 555, 504-523 (2001)

5. Takahashi, R. \& Chiba, T. Gravitational lens statistics and the density profile of dark halos. Astrophys. J. 563, 489-496 (2001).

6. Oguri, M. Constraints on the baryonic compression and implications for the fraction of dark halo lenses. Astrophys. J. 580, 2-11 (2002).

7. Navarro, J. F., Frenk, C. S. \& White, S. D. M. A universal density profile from hierarchical clustering. Astrophys. J. 490, 493-508 (1997).

8. Maoz, D., Rix, H., Gal-Yam, A. \& Gould, A. Survey for large-image separation lensed quasars. Astrophys. J. 486, 75-84 (1997).

9. Ofek, E. O., Maoz, D., Prada, F., Kolatt, T. \& Rix, H. A survey for large-separation lensed FIRST quasars. Mon. Not. R. Astron. Soc. 324, 463-472 (2001).

10. Phillips, P. M. et al. The JVAS/CLASS search for 6-arcsec to 15-arcsec image separation lensing. Mon. Not. R. Astron. Soc. 328, 1001-1015 (2001).

11. Zhdanov, V. I. \& Surdej, J. Quasar pairs with arcminute angular separations. Astron. Astrophys. 372, $1-7$ (2001).

12. Kochanek, C. S. et al. CASTLES survey. 〈http://cfa-www.harvard.edu/castles/〉 (2003).

13. Walsh, D., Carswell, R. F. \& Weymann, R. J. $0957+561$ A, B - Twin quasistellar objects or gravitational lens? Nature 279, 381-384 (1979).

14. Colley, W. N., Tyson, J. A. \& Turner, E. L. Unlensing multiple arcs in 0024+1654: Reconstruction of the source image. Astrophys. J. 461, L83-L86 (1996).

15. Turner, E. L., Ostriker, J. P. \& Gott, J. R. III The statistics of gravitational lenses-the distributions of image angular separations and lens redshifts. Astrophys. J. 284, 1-22 (1984).
16. York, D. G. et al. The Sloan Digital Sky Survey: Technical summary. Astron. J. 120, 1579-1587 (2000). 17. Stoughton, C. et al. Sloan Digital Sky Survey: Early data release. Astron. J. 123, 485-548 (2002). 18. Gunn, J. E. et al. The Sloan Digital Sky Survey photometric camera. Astron. J. 116, 3040-3081 (1998) 19. Pier, J. R. et al. Astrometric calibration of the Sloan Digital Sky Survey. Astron. J. 125, 1559-1579 (2003).

20. Hogg, D. W., Finkbeiner, D. P., Schlegel, D. J. \& Gunn, J. E. A photometricity and extinction monitor at the Apache Point Observatory. Astron. J. 122, 2129-2138 (2001).

21. Smith, J. A. et al. The u' $\mathrm{g}^{\prime} \mathrm{r}^{\prime} \mathrm{i}^{\prime} \mathrm{z}^{\prime}$ standard-star system. Astron. J. 123, 2121-2144 (2002)

22. Fukugita, M. et al. The Sloan Digital Sky Survey photometric system. Astron. J. 111, 1748-1756 (1996).

23. Blanton, M. R. et al. An efficient targeting strategy for multiobject spectrograph surveys: The Sloan Digital Sky Survey "tiling" algorithm. Astron. J. 125, 2276-2286 (2003)

24. Richards, G. T. et al. Spectroscopic target selection in the Sloan Digital Sky Survey: The quasar sample. Astron. J. 123, 2945-2975 (2002).

25. Oguri, M., Taruya, A., Suto, Y. \& Turner, E. L. Strong gravitational lensing time delay statistics and the density profile of dark halos. Astrophys. J. 568, 488-499 (2002).

26. Kashikawa, N. et al. FOCAS: The Faint Object Camera and Spectrograph for the Subaru Telescope. Publ. Astron. Soc. Jpn 54, 819-832 (2002).

27. Cao, L., Wei, J.-Y. \& Hu, J.-Y. High X-ray-to-optical flux ratio RASS-BSC sources. I. The optical identification. Astron. Astrophys. Suppl. 135, 243-253 (1999).

28. Keeton, C. R. Computational methods for gravitational lensing. Preprint at 〈http://xxx.lanl.gov/astro$\mathrm{ph} / 0102340\rangle$ (2001).

29. Oke, J. B. et al. The Keck Low-Resolution Imaging Spectrometer. Publ. Astron. Soc. Pacif. 107, 375-385 (1995).

30. Miyazaki, S. et al. Subaru prime focus camera-Suprime-Cam. Publ. Astron. Soc. Jpn 54, 833-853 (2002)

Acknowledgements Funding for the creation and distribution of the SDSS Archive has been provided by the Alfred P. Sloan Foundation, the Participating Institutions, the National Aeronautics and Space Administration, the National Science Foundation, the US Department of Energy, the Japanese Monbukagakusho, and the Max Planck Society. The SDSS website is http://www.sdss.org/. The SDSS is managed by the Astrophysical Research Consortium (ARC) for the Participating Institutions. The Participating Institutions are The University of Chicago, Fermilab, the Institute for Advanced Study, The Japan Participation Group, The Johns Hopkins University, Los Alamos National Laboratory, the Max-Planck-Institute for Astronomy (MPIA), the Max-Planck-Institute for Astrophysics (MPA), New Mexico State University, University of Pittsburgh, Princeton University, the United States Naval Observatory, and the University of Washington. This Letter is based in part on data collected at the Subaru telescope, which is operated by the National Astronomical Observatory of Japan, W. M. Keck Observatory, which is operated as a scientific partnership among the California Institute of Technology, the University of California, and the National Aeronautics and Space Administration, and the Apache Point Observatory (APO) 3.5-m telescope, which is owned and operated by the Astrophysical Research Consortium. Part of this work was performed under the auspices of the U.S. Department of Energy at the University of California Lawrence Livermore National Laboratory.

Competing interests statement The authors declare that they have no competing financial interests.

Correspondence and requests for materials should be addressed to N.I. (inada@utap.phys.s.u-tokyo.ac.jp).

\section{Subatomic movements of a domain wall in the Peierls potential}

\section{K. S. Novoselov ${ }^{1}$, A. K. Geim ${ }^{1}$, S. V. Dubonos ${ }^{3}$, E. W. Hill ${ }^{2}$ \& I. V. Grigorieva ${ }^{1}$}

${ }^{1}$ Department of Physics, ${ }^{2}$ Department of Computer Sciences, University of Manchester, Manchester M13 9PL, UK

${ }^{3}$ Institute for Microelectronics Technology, 142432 Chernogolovka, Russia

The discrete nature of crystal lattices plays a role in virtually every material property. But it is only when the size of entities hosted by a crystal becomes comparable to the lattice period-as occurs for dislocations ${ }^{1-3}$, vortices in superconductors ${ }^{4-6}$ and domain walls ${ }^{7-9}$-that this discreteness is manifest explicitly. The associated phenomena are usually described in terms of a background Peierls 'atomic washboard' energy potential, which was first introduced for the case of dislocation motion ${ }^{1,2}$ in the 1940s. This concept has subsequently been invoked in many situations to describe certain features in the bulk behaviour of materials, but has to date eluded direct detection and experimental scrutiny at a microscopic level. Here we report observations of 